\title{
In Situ Calibration for Quantitative Infrared Thermography
}

\author{
M. Martiny, R. Schiele, M. Gritsch, A. Schulz, S. Wittig \\ Institut für Thermische Strömungsmaschinen, Universität Karlsruhe (TH), 76128 Karlsruhe, Germany
}

\begin{abstract}
This paper presents a method to correct temperatures obtained by infrared thermography using thermocouples as reference temperatures. The raw signal values of the infrared detector are compared to the temperatures measured with thermocouples. The relation between raw thermal value and body temperature includes three parameters which allow to determine a best-fit approximation between detector signal and body temperature. The resulting non-linear system of equations is solved numerically using the Levenberg-Marquard procedure. These parameters are then used to calculate the corrected temperatures of the body.
\end{abstract}

\section{Introduction}

Infrared thermography is a powerful method for temperature measurement in turbomachinery research. At the 'Institut für Thermische Strömungsmaschinen' at the 'Universität Karlsruhe' the infrared camera has been used in numerous investigations on heat transfer phenomena. So far, the results achieved were mainly used for qualitative reviews of complex surface temperature distributions. To allow for any quantitative analyses the data have to undergo some further correction or calibration procedure. This procedure has to account for many different effects (e.g. reflected radiation, gas radiation, translucency of the window) which have an impact on the thermography results. In most experimental set-ups matching real engine conditions, these effects are difficult to quantify. Therefore, any correction procedure on a pure theoretical basis is not very promising. Better accuracy can be achieved with a case specific calibration of the thermography system which is obtained experimentally.

Various authors have proposed calibration procedures for thermography systems which rely on calibration experiments. Among them Koschel et al. [1] used an external system to calibrate a pyrometer which was used to measure the temperatures on the surface of turbine blades. In this way the influence of the observation angle and the distance between blade surface and detector on the IR-signal could be studied precisely. Nevertheless, the surface emissivity could not be determined accurately and reflection effects could not be simulated in the calibration setup. Carlomagno et al. [2] used a little black body to calibrate an IR-camera under real test conditions. The black body was placed in the test rig at the location at which the surface temperature had to be determined later on. The calibration parameters obtained with the black body were then used in the surface temperature measurements with an additional correction for gray body. This method can only be applied if the test surface can be dealt as a gray body, i.e. its emissivity is independent of temperature, and if the test section allows for the insertion of a black body. Reflected radiation, which occurs in the case of any gray body, cannot be taken into account. Meyers at al. [3] performed temperature measurements on film cooled combustor walls. During the heating-up of the combustor, temperature data were recorded from both the infrared scanner and from the embedded thermocouples. These data were then paired and used for a best fit approximation for the calibration parameters of the camera. Thus, all temperature dependent properties of the system are taken into account. Nevertheless thermal image recording during the heating-up period leads to slightly shifted operating conditions between calibrating and testing. The calibration method presented in this paper uses a similar approach but thermocouple temperature and thernial image recording takes place at steady state and the 'best-fit' approximation includes three parameters instead of two for higher accuracy. 


\section{Temperature Measurement Technique}

\subsection{Theoretical Aspects}

The relation between the spectral infrared radiation and the temperature of a black body is given by the law of Planck (Fig. 1):

$$
q_{s}^{\prime \prime}(\lambda)=\frac{c_{1} \lambda^{-5}}{e^{\frac{c_{2}}{\lambda T}}-1}
$$

$c_{1}$ and $c_{2}$ are physical constants. Usually technical surfaces are not equivalent to a black body. The radiation is then calculated by

$$
q_{g}^{\prime \prime}(\lambda)=\varepsilon(\lambda) \cdot q_{s}^{\prime \prime}(\lambda)=\varepsilon(\lambda) \cdot \frac{c_{1} \lambda^{-5}}{e^{\frac{c_{2}}{\lambda T}}-1}
$$

where $\varepsilon$ represents the emissivity of the radiating surface. If the spectral irradiance is measured and the emissivity of the object is known then a corresponding surface temperature can be calculated:

$$
T=\frac{c_{2} / \lambda}{\ln \left(\frac{\varepsilon(\lambda) \cdot c_{1} \cdot \lambda^{-5}}{\dot{q}_{g}^{\prime \prime}}+1\right)}
$$

\subsection{Description of Infrared Scanner System}

The system used in the present investigations is an AGEMA Thermovision 870 infrared scanner with a thermoelectrically-cooled mercury-cadmium-telluride detector, sensitive to infrared radiation in the spectral band of 2.0 to $5.0 \mu \mathrm{m}$ (Fig. 1). Thermal radiation from the surface is focused through lenses and a rotating prism on the detector. Being exposed to radiation, the detector generates a voltage which can be used to calculate an object temperature. Since the scanner is integrally sensitive in a certain spectral band only and the emissivity is usually an unknown function of temperature and wavelength, the theoretical relation is replaced by a semiempirical equation:

$$
I=\frac{R}{e^{8 / T}-F}
$$

The structure of this formula is similar to Eq. 1. R, B and $F$ are parameters which have to be determined to obtain the 'best-fit' relation between I and T. I, which is called 'thermal value', is measured on an arbitrary scale and corresponds to the radiation received by the detector.

The overall radiation detected by the camera can be divided into three components (Fig. 2). In a general situation the object has an emissivity lower than unity and the transmissivity of the atmosphere between object and camera is $\tau_{\text {atm }}$ so that the thermal value corresponding to the object is $I_{1}$ (see Eq. 5). The atmosphere between camera and object may emit (e.g. combustion gas) a certain amount of radiation which will be received by the detector $\left(\mathrm{I}_{2}\right)$. As the object is not a black body, radiation of the surroundings is reflected on the object's surface. Assuming an emissivity of unity for the surroundings this radiation contributes to the thermal value as given in Eq. 5 for $I_{3}$. The total thermal value is then expressed by:

$$
I_{\text {tot }}=I_{1}+I_{2}+I_{3}=\tau_{a t m} \cdot \varepsilon_{o b j} \cdot I_{o b j}+\left(1-\tau_{a t m}\right) \cdot I_{a t m}+\tau_{a t m} \cdot\left(1-\varepsilon_{o b j}\right) \cdot I_{s}
$$

To calculate the temperature of the object Eq. 4 is rewritten:

$$
T_{o b j}=\frac{B}{\ln \left(R / I_{o b j}+F\right)}
$$

The thermal value corresponding to the object can be calculated from Eq. 5: 


$$
l_{o b j}=\frac{l_{\text {tot }}}{\varepsilon_{o b j} \cdot \tau_{o b j}}-\frac{\left(1-\varepsilon_{o b j}\right)}{\varepsilon_{o b j}} \cdot I_{s}-\frac{\left(1-\tau_{a t m}\right)}{\tau_{a t m} \cdot \varepsilon_{o b j}} \cdot I_{a t m}
$$

Knowing the temperature of the atmosphere and of the surroundings $l_{\mathrm{s}}$ and $\mathrm{I}_{\mathrm{atm}}$ can be calculated using Eq. 4. The default parameters $\mathrm{R}, \mathrm{B}$ and $\mathrm{F}$ are determined by the manufacturer in a black body experiment which is set up in a way so that atmospheric radiation and radiation from surroundings are negligible.

This procedure is certainly a good approximation for a general measurement situation. However, for accurate quantitative measurements under test conditions representative for modern machinery, e.g. turbomachinery engines, a more sophisticated calibration procedure is needed.

\subsection{Description of the Correction Procedure}

As mentioned above, the correction procedure is based on thermocouple temperature values and a corresponding thermal image recorded simultaneously under steady state test conditions. These thermocouple temperature data are used as reference values for the calibration. It is, therefore, very important to position the thermocouples on the object surface in a way to cover the whole temperature range of interest.

During the thermal image recording all user-defined parameters (e.g. temperatures of the atmosphere and of the surroundings, emissivities, transmittance of the atmosphere) are determined as well as possible to get reasonable results without changing the default values for $R$, $B$ and $F$. After completion of the measurements, the difference between a reference temperature and the temperature obtained from the IR-image at a point ' $i$ ' is given by:

$$
\mathrm{X}_{i}=T_{i}-\frac{B}{\ln \left(R / I_{i}+F\right)}
$$

In calculating the error squares the following expression is obtained for each reference point:

$$
\mathrm{X}_{i}^{2}=T_{i}^{2}-2 \cdot \frac{B \cdot T_{i}}{\ln \left(R / I_{i}+F\right)}+\frac{B^{2}}{\left[\ln \left(R / I_{i}+F\right)\right]^{2}}
$$

For minimization of the error squares the following conditions have to be fulfilled:

$$
f_{1}=\frac{\partial\left(\sum_{i=1}^{n} X_{i}^{2}\right)}{\partial R}=0 ; f_{2}=\frac{\partial\left(\sum_{i=1}^{n} X_{i}^{2}\right)}{\partial B}=0 ; f_{3}=\frac{\partial\left(\sum_{i=1}^{n} X_{i}^{2}\right)}{\partial F}=0
$$

This system of 3 non-linear equations for $R, B$ and $F$ has to be solved by an adequate numerical algorithm. Since the quality of the thermal image, i.e. the starting solution, can differ significantly from case to case, a very robust solver is required. An elegant method combining the advantages of the inverse Hessian method and of the steepest descent method is the Levenberg-Marquardt procedure. Details are described in [4] and will not be discussed here. Once the system is solved, the new values for $\mathrm{R}, \mathrm{B}$ and $\mathrm{F}$ are entered in $\mathrm{Eq} .6$ and a new, more accurate temperature field is obtained. The capability of this procedure will be demonstrated on the basis of three different experimental set-ups with varying test conditions.

\section{Results and Discussion}

At the 'Institut für Thermische Strömungsmaschinen' film cooling investigations are performed at different scales ranging from the detailed characterization of the cooling film of a single-hole with expanded exit over the analyses of full-coverage film cooling combustor walls with an array of holes and finally to the investigation of a real film cooled turbine blade.

\subsection{Film Cooling holes with expanded exits}

Modern airfoils are equipped with complex cooling regimes combining various convection cooling methods on the inner surface and film cooling on the outer surface. Recently, attention has 
been drawn to shaping the exit of the film cooling holes to reduce the momentum of the exiting jet preventing jet separation from the blade surface. The present study focuses on the detailed investigation of the effect of hole geometry on the flowfield and the film cooling effectiveness in the vicinity of the film-cooling hole. For this purpose a new experimental setup has been built [9]. Figure 3 shows one of the test-plates which are used in the investigations. The hot gas and the coolant air are at temperatures of $530 \mathrm{~K}$ and $290 \mathrm{~K}$, respectively. The distance between camera and test-plate is $1000 \mathrm{~mm}$. Using a camera lens with a field of view of $7^{\circ}$ a spatial resolution of $0.7 \mathrm{~mm} / \mathrm{pixel}$ is obtained. As reference, 15 thermocouples are embedded in the surface. While having a considerable difference between reference temperatures and IR-image for the raw data the RMS-value in temperature is reduced to $0.41 \mathrm{~K}$ by applying the calibration procedure.

\subsection{Effusion Cooling of Combustor Liners}

Effusion cooling is a promising concept to meet the requirements (high cooling effectiveness with low cooling air consumption) of modern low-emission combustion chambers. The cooling air is blown through the perforated wall and exits on the hot gas side forming a cooling film to protect the liner from the hot combustion gas. Cooling effectiveness and heat transfer coefficient distribution are of major interest in designing the liner geometry [5]. Figure 5 presents a schematic view of the experimental set-up. A detailed description of the experimental investigations is given in [5]. The distance between the object surface and the camera is about $300 \mathrm{~mm}$. The field of view is $40^{\circ}$ resulting in a resolution of $1.56 \mathrm{~mm} / \mathrm{pixel}$. The object surface is observed through a sapphire window placed in the channel lid. The main flow is heated up to a temperature of $570 \mathrm{~K}$ with an electrical heater. There are no combustion products influencing the infrared measurements. The atmosphere between channel lid and camera lens is at ambient. The surface of the test plate is painted with a special velvet coating with a temperature independent emissivity of $\varepsilon=0.95$ to obtain excellent radiation conditions [6]. Fourteen embedded thermocouples are within the field of view at positions between the effusion holes (Fig. 5). As the cooling efficiency is varying with downstream distance the surface temperature is varying also and the thermocouple data cover the whole interesting temperature range. Figure 6 presents the relative error distribution obtained with the default values for $R, B$ and $F$ and the calibration values specific to the test case. The root mean square in temperature could be reduced from $7,5 \mathrm{~K}$ to $2,6 \mathrm{~K}$.

\subsection{Film-Cooled Gas Turbine Guide Vane}

Accuracy and resolution of the heat transfer measurement technique, which has been used in various test rigs at the 'Institut für Thermische Strömungsmaschinen' on convectively cooled turbine blades (e.g. Schiele et al. [7], Wittig et al. [8]), are mainly dependent upon the precise temperature measurement on the blade surface. So far thermocouples were used to determine the surface temperatures. By employing the infrared scanner the method could be extended to the investigation of film cooled blades. In the experimental setup shown in Figure 7 camera and blade surface are about $650 \mathrm{~mm}$ apart. Looking through a sapphire glass window with an objective having a field of view of $7^{\circ}$, a resolution of $0.5 \mathrm{~mm} / \mathrm{pixel}$ is obtained. Again the velvet coating described in [6] is used for temperature and angle independent emissivity. The blade surface is heated by internal water pipe flows, resulting in surface temperatures ranging from $290 \mathrm{~K}$ to $340 \mathrm{~K}$. The main flow has a temperature of about $335 \mathrm{~K} .19$ thermocouples are embedded in the surface. By employing the calibration method described, the RMS-value in temperature could be reduced from $3.24 \mathrm{~K}$ to a marginal value of $0.21 \mathrm{~K}$.

\section{Conclusions}

The present paper describes a new calibration method for infrared thermography data. The method relies on the comparison of the so-called thermal value of the infrared detector with reference temperatures. By solving a system of 3 non-linear equations the 3-parametric relation between thermal value and temperature is optimized so that the differences between reference 
temperatures and temperatures from the IR-image are minimized. The method is applied to three different experimental set-ups under varying test conditions. The results demonstrate the capability of the new calibration procedure.

\section{Acknowledgments}

The work reported was partly funded within the framework of the 'AG TURBO' by the 'Bundesministerium für Bildung, Wissenschaft, Forschung und Technologie' and the 'BMW Rolls-Royce GmbH' under contract 03226760B and the 'Motoren- und Turbinen-Union München $\mathrm{GmbH}$ ' under contract $0326821 \mathrm{H}$ and also within the Brite-Euram project AEROCT92-0044. Authors are responsible for the content of the paper.

\section{REFERENCES}

[1] KOSCHEL (W.), SALDEN (D.), HOCH (T.).- Turbine Rotor Blade Measurements Using Infrared Pyrometry. AGARD CP-399, Pennsylvania, USA,1986,p. 18-1/18-16

[2] ASTARITA (T.), CARDONE (G.), CARLOMAGNO (G.). - Heat Transfer and Flow Visualization Around a 180 deg Turn in a rectangular Channel. Proceeding HTD-Vol. 318, San Francisco, USA, 1995

[3] MEYERS (G.), VAN DER GEEST (J.), SANBORN (J.), DAVIS (F.).- Comparison of Advanced Cooling Concepts Using Color Thermography. AIAA-85-1289, Monterey, USA, 1985

[4] PRESS (W.), FLANNERY (B.), TEUKOLSKY (S.), VETTERLING (W.)-Numerical Recipes. Cambridge University Press, 1987

[5] MARTINY (M.), SCHULZ (A.) and WITTIG (S.).-Full-Coverage Film Cooling Investigations: Adiabatic Wall Temperatures and Flow Visualization. 95-WA/HT-4,San Francisco, USA, 1995

[6] LOHRENGEL (J.).-Gesamtemissionsgrad von Schwärzen. Wärme- und Stoffübertragung, Vol. 90, S. 157-163, 1968 [in German]

[7] SCHIELE (R.), SIEGER (K.), SCHULZ (A.), WITTIG (S.).- Heat Transfer Investigations on a Highly Loaded, Aerothermally Designed Turbine Cascade. In: F. Billig (ed.), 12th Int. Symposium on Air Breathing Engines, pp. 1091-1101, Melbourne, Australia, 1995

[8] WITTIG (S.), SCHULZ (A.) and BAUER (H.-J.).-Effects of Wakes on the Heat Transfer in Gas Turbine Cascades. AGARD-CP-390, 1985

[9] WITTIG, (S.), SCHULZ (A.), GRITSCH (M.), THOLE (K.A.).- Transonic Film-Cooling Investigations: Effects of Hole Shapes and Orientations. ASME Paper 96-GT-222, Birmingham, UK, 1996

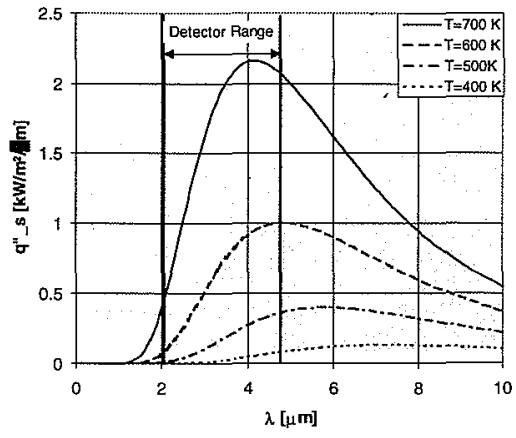

Fig. 1: Spectral radiation energy distribution for a black body

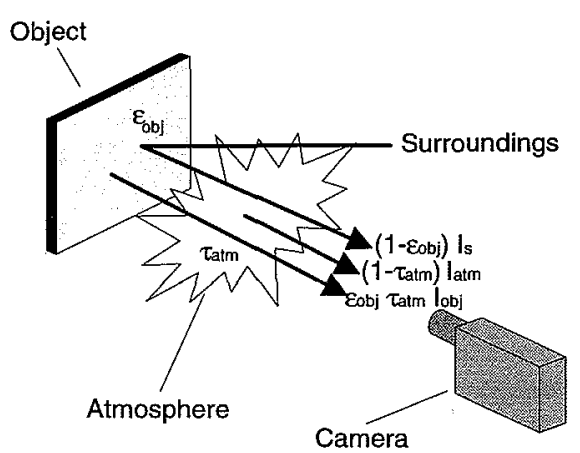

Fig. 2: Radiation conditions in the general measurement situation 

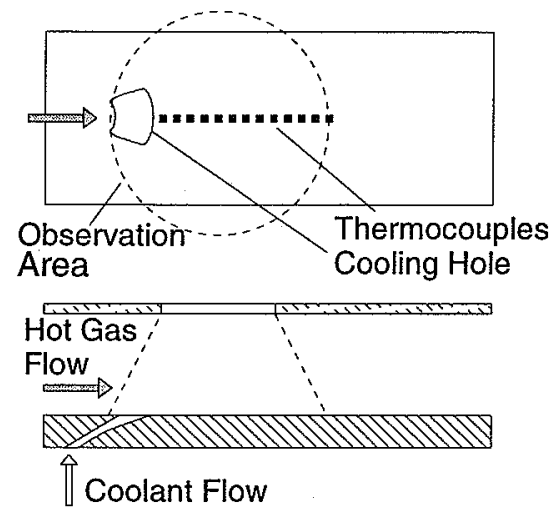

Fig. 3: Experimental set-up for the laidbackfanshaped cooling hole

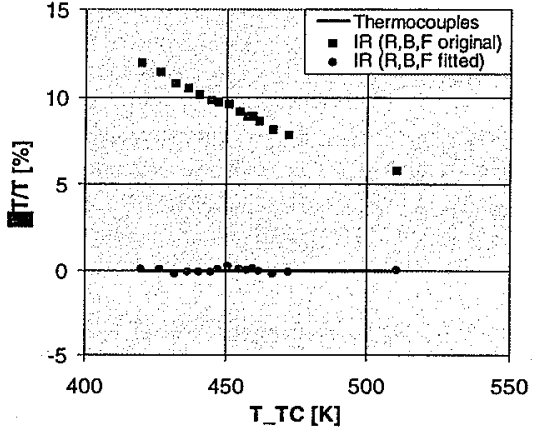

Fig. 4: Relative error in temperature for the laid back-fanshaped cooling hole
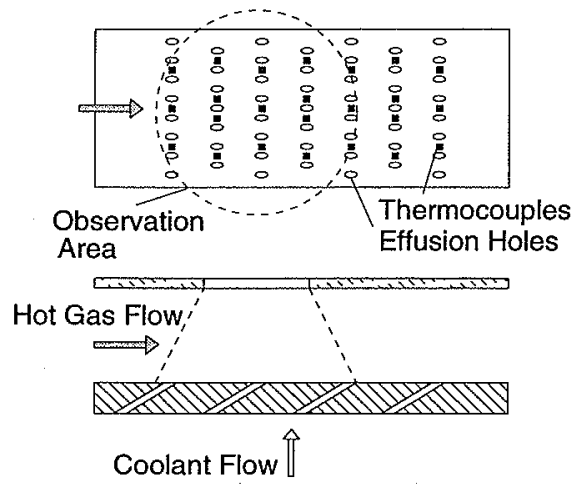

Fig. 5: Experimental set-up for the effusioncooled testplate

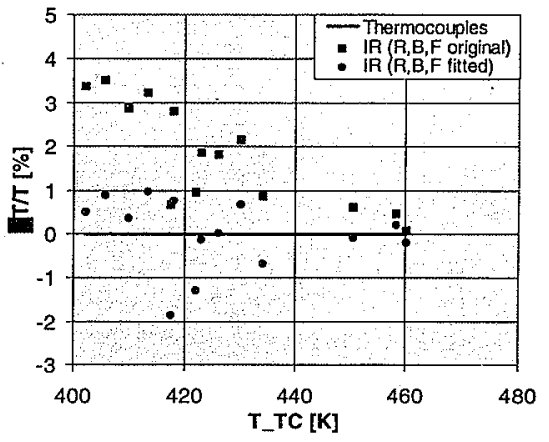

Fig. 6: Relative error in temperature for the effusion-cooled testplate

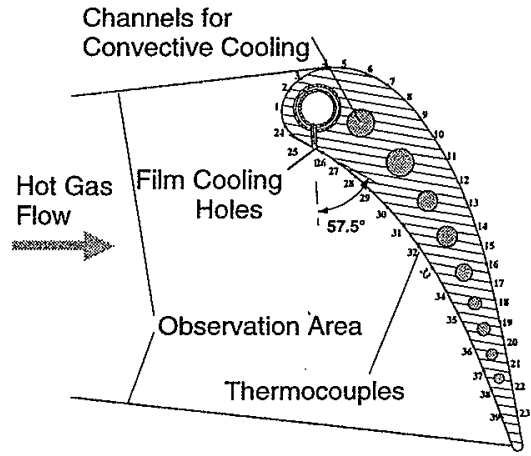

Fig. 7: Experimental set-up for the film-cooled guide vane

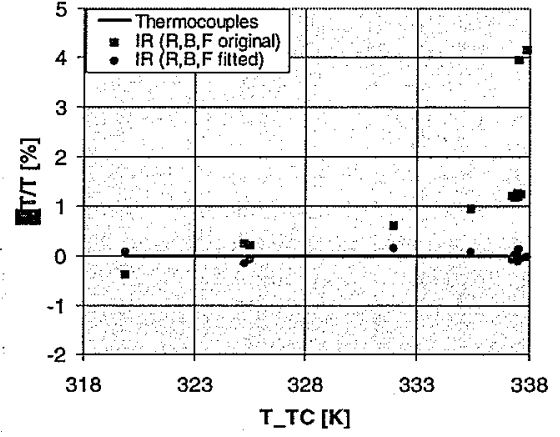

Fig. 8: Relative error in temperature for the filmcooled blade 